\title{
Mast Cell-dependent Mesenteric Afferent Activation by Mucosal Supernatant From Different Bowel Segments of Guinea Pigs With Post-infectious Irritable Bowel Syndrome
}

\author{
Jun Song, Lei Zhang, Tao Bai, Wei Qian, Rui Li and Xiaohua Hou* \\ Division of Gastroenterology, Wuhan Union Hospital, Huazhong University of Science and Technology, Wuhan, China
}

\begin{abstract}
Background/Aims
Mesenteric afferent nerves (MANs) play a pivotal role in the visceral-nociceptive perception. Inappropriate activation of MANs may be involved in the pathogenesis of post-infectious irritable bowel syndrome (PI-IBS). However, the underlying mechanisms remain unclear. We assessed the effects of mucosal mediators from different bowel segments of guinea pigs with PI-IBS on MAN firing and the role of mast cells.
\end{abstract}

\section{Methods}

PI-IBS was induced in guinea pigs by Trichinella spiralis infection. Inflammation in terminal ileum, proximal and distal colon was scored with hematoxylin-eosin staining, and mast cell infiltration was assessed with immunofluorescence. We determined the effects of supernatant extracted from the mucosa of different bowel segments of PI-IBS on MANs activity, and assessed the role of mast cells in this process.

\section{Results}

Eight weeks after infection, intestinal inflammation resolved, whereas mast cell numbers increased significantly in terminal ileum and proximal colon $(P<0.05)$ compared with findings in controls. Mucosal supernatant from different bowel segments of PI-IBS models, but not from controls, significantly enhanced the frequency of MAN firing (terminal ileum $41.01 \pm 7.60 \mathrm{~Hz} \mathrm{vs}$. $26.55 \pm 0.67 \mathrm{~Hz}, P=0.001$; proximal colon $45.90 \pm 11.20 \mathrm{~Hz}$ vs. $30.88 \pm 6.92 \mathrm{~Hz}, P=0.002$; distal colon $48.25 \pm 9.70$ $\mathrm{Hz}$ vs. $29.47 \pm 6.13 \mathrm{~Hz}, P<0.001$ ). In addition, the excitatory effects were inhibited by mast cell stabilizer Nasmil (terminal ileum, $32.71 \pm 2.52 \mathrm{~Hz}, P=0.030$; proximal colon, $30.94 \pm 4.44 \mathrm{~Hz}, P=0.002$; distal colon, $27.15 \pm 5.83 \mathrm{~Hz}, P<$ $0.001)$.

\section{Conclusions}

Supernatant from the intestinal mucosa of different bowel segments of PI-IBS models markedly enhanced the MAN firing in

Received: August 25, 2014 Revised: December 8, 2014 Accepted: December 14, 2014

(c) This is an Open Access article distributed under the terms of the Creative Commons Attribution Non-Commercial License (http://creativecommons. org/licenses/by-nc/3.0) which permits unrestricted non-commercial use, distribution, and reproduction in any medium, provided the original work is properly cited.

*Correspondence: Xiaohua Hou, MD, PhD

Division of Gastroenterology, Union Hospital, Tongji Medical College, Huazhong University of Science and Technology,1277 Jiefang Road, Wuhan 430022, China

Tel: +86-027-85726057, Fax: +86-027-85726057, E-mail: houxh@medmail.com.cn

Financial support: This study was supported by a grant from The National Natural Science Foundation of China (NSFC) (Grant No. 81070300 and 81200271).

Conflicts of interest: None.

Author contributions: Xiaohua Hou designed the research study; Jun Song, Wei Qian, Lui Li, and Lei Zhang performed the experiment; Tao Bai analyzed the data; Jun Song drafted the manuscript.

ORCID: Xiaohua Hou, http://orcid.org/0000-0002-4777-7920. 
a mast cell-dependent manner, indicating that mast cell-mediated MAN activation plays an important role in the pathogenesis of PI-IBS.

\section{(J Neurogastroenterol Motil 2015;21:236-246)}

\section{Key Words}

Hyperalgesia; Immunity; Irritable bowel syndrome; Mast cell; Visceral afferents

\section{Introduction}

Irritable bowel syndrome (IBS) is a functional gastrointestinal disorder commonly seen in the gastroenterology clinic. IBS prevalence among the general population in Western societies is about $10 \%$ to $20 \%$, ${ }^{1}$ however, the underlying mechanisms of IBS are still unknown. Recent studies suggested that a proportion of patients with IBS began with acute gastroenteritis; which is known as post-infectious IBS (PI-IBS). ${ }^{2}$ In Western countries, about 3.7\% to $36 \%$ of patients develop IBS after acute gastrointestinal infection. ${ }^{3}$ The possible mechanisms of PI-IBS include mucosal injury and inflammation, ${ }^{4}$ increased intestinal permeability, ${ }^{5}$ mast cell hyperplasia, ${ }^{6}$ and enteric nerve alteration. ${ }^{7,8}$

Abdominal pain is the most predominant and distressing symptom of PI-IBS. ${ }^{9}$ Visceral hypersensitivity, which is generally considered to be a hallmark of IBS with lowered thresholds for pain and increased intensity of sensations, was responsible for the symptom. ${ }^{10}$ It is known that visceral sensation is regulated by the nervous system at different levels: the enteric nervous system (ENS), autonomic nervous system, and central nervous system. Abundant evidence has confirmed that the dysregulation of nerves related to visceral sensation plays an important role in IBS. For example, we found that synaptic plasticity in ENS of PI-IBS model rats infected with Trichinella spiralisis associated with visceral sensitivity. ${ }^{7}$ Dorsal root ganglia neuron excitability was increased significantly in the PI-IBS model. ${ }^{11}$ Others showed through functional magnetic imaging and positron emission tomography studies that brain areas involved in visceral sensation processing, such as the prefrontal cortex and cingulate cortex, were activated abnormally in IBS patients. ${ }^{12}$ As an important component of brain- gut axis, ANS was also disordered in patients with IBS, indicated by increased sympathetic activity and decreased parasympathetic activity. ${ }^{13,14}$

As a part of peripheral component, mesenteric afferent nerves (MANs) dominate the gut and detect intestinal microenvironmental changes directly. ${ }^{15}$ It collects information from the gut and transmits the information to central nervous system via the vagal or splanchnic nerve. ${ }^{16}$ Therefore, MANs occupy an important position in visceral perception, especially nociceptive stimuli and inappropriate activation of MANs may lead to abdominal pain. However, the role of MANs in PI-IBS is still not well understood.

In addition, low-grade inflammation of rectal mucosa has been confirmed in PI-IBS. ${ }^{3,17}$ However, whether bowel segments other than the rectum are inflamed in PI-IBS is unclear. Furthermore, mast cells play an important role in local immune dysregulation of PI-IBS. In animal models with visceral hypersensitivity and in patients with IBS, an increased number of mast cells were found in the intestinal tract compared with healthy controls. Interestingly, an estimated $70 \%$ of intestinal mucosal mast cells were in direct contact with nerve fiber, and another $20 \%$ were located within 2 $\mu \mathrm{m} .{ }^{18}$ This finding provides a basis for neuro-immune interaction in the gut, which may be involved in the development of IBS. ${ }^{19}$ However, the specific mechanism of mast cells in this process is unknown.

We hypothesize that low-grade inflammation occurs in different bowel segments of PI-IBS and activates MANs, and that mast cells play a major role in this process. To test our hypothesis and elucidate whether MANs are involved in the pathogenesis of PI-IBS, we examined MAN activity induced by mucosal extract from different intestinal segments from PI-IBS models and controls and assessed the role of mast cells in this process.

\section{Materials and Methods}

\section{Animals and Trichinella spiralis Infection}

All experimental procedures were approved by the animal care and use committee of the Union Hospital of Tongii Medical College. T. spiralis infection is an established animal model for PI-IBS in rodents. ${ }^{20-22}$ Outbred, adult female guinea pigs weighing $303 \pm 34 \mathrm{~g}$ were each orally inoculated with $T$. spiralis muscle-stage larvae in $0.2 \mathrm{~mL}$ phosphate buffered saline. The control 
group was administered with $0.2 \mathrm{~mL}$ phosphate buffered saline without T. spiralis larvaes. Each group contained 10 guinea pigs. All animals were fed with standard guinea pig chow supplemented with vitamin $\mathrm{C}$ and had free access to water. Eight weeks after inoculation with $T$. spiralis, the guinea pigs were used for the experiments described below.

\section{Histology and Inflammation Scores}

$\mathrm{H} \& \mathrm{E}$ staining of terminal ileum, proximal colon, and distal colon specimens was performed using standard techniques. The severity of inflammation was graded with a scale previously described by Al-Chaer et $\mathrm{al}^{23}$ as follows: $1+$, mild (infiltration of a low number of neutrophils in the lamina propria and little or no interstitial edema); $2+$, moderate (infiltration of moderate numbers of neutrophils in the lamina propria and moderate interstitial edema); and $3+$, severe (diffuse infiltration of moderate to large numbers of neutrophils in the lamina propria and severe interstitial edema). The severity of mucosal inflammation was scored blindly by 2 investigators, and a mean score was obtained.

\section{Immunofluorescence and Mast Cell Counts}

The intestinal specimens were fixed with $4 \%$ paraformaledehyde, embedded in paraffin, cut and processed for immunofluorescence analysis. Briefly, the specimens were blocked with $5 \%$ bovine serum albumin (containing $0.3 \%$ Triton X-100), and then incubated with mouse anti-mast cell tryptase monoclonal antibody (1:250; Abcam plc, Cambridge, UK) as the primary antibody overnight at $4^{\circ} \mathrm{C}$, and then with Alexa Fluor 488 donkey anti-mouse $\operatorname{IgG}(\mathrm{H}+\mathrm{L})$ (1:200; Invitrogen, Carlsbad, CA, USA) as the secondary antibody for 1 hour at room temperature. Finally, nuclei were stained with DAPI $(1 \mu \mathrm{g} / \mathrm{mL}$; Beyotime Biotech, Jiangsu, China). Sections were viewed using a confocal laser scanning microscope (Nikon, Tokyo, Japan) with an excitation wavelength appropriate for Alexa Fluor (488 nm). Images were captured and analyzed using NIS Elements Viewer Software (Nikon). Mast cells, namely tryptase-positive cells, were quantified by the same researcher without knowledge of the groupings. Ten contiguous non-overlapping fields per immunostained slide from each group were counted and the results were expressed as mast cells per high power field (mast cells/hpf).

\section{Tissue Preparation and Recording of Mesenteric Afferent Nerve Firing}

The method of tissue preparation was described previously. ${ }^{24}$ In brief, the guinea pigs were anesthetized with $2 \%$ pentobarbital sodium $(80 \mathrm{mg} / \mathrm{kg})$, and then the proximal jejunum in which the mesenteric vessel had no bifurcation was removed and bathed into ice-cold and oxygenated $\left(95 \% \mathrm{O}_{2}+5 \% \mathrm{CO}_{2}\right)$ Krebs solution (120 mM NaCl, $5.9 \mathrm{mM} \mathrm{KCl}, 1.2 \mathrm{mM} \mathrm{NaH}{ }_{2} \mathrm{PO}_{4}, 1.2 \mathrm{mM}$ $\mathrm{MgSO}_{4}, 15.4 \mathrm{mM} \mathrm{NaHCO} 3,2.5 \mathrm{mM} \mathrm{CaCl}_{2}$, and $11.5 \mathrm{mM}$ glucose; $\mathrm{pH}$ 7.3-7.4). Each segment was securely attached in a recording chamber at either end to an input and outlet port. The input port was connected to a syringe pump that allowed continuous intraluminal perfusion of Krebs solution through the segments while the outlet port was open. The mesenteric bundle was pinned onto the Sylgard base of the recording chamber. The mesenteric nerve was carefully dissected under a dissecting microscope (World Precision Instruments Inc, Sarasota, FL, USA). Then the mesenteric nerve was suspended on a recording electrode, and the connective tissue was suspended on a reference electrode. The organ chamber was circulated with oxygenated Krebs solution maintained at $33-34^{\circ} \mathrm{C}$ at a flow of $10 \mathrm{~mL} / \mathrm{hr}$. Each segment was immersed in oxygenated Krebs solution containing $1 \mu \mathrm{M}$ nifedipine to eliminate the influence of smooth muscle spontaneous contraction and the solution was circulated at a flow of $10 \mathrm{~mL} / \mathrm{hr}$. Mesenteric nerve activity was recorded with a Neurolog headstage (PowerLab 4/26; ADInstruments, New South Wales, Australia) and electrical signal which was amplified (Model 1800 Microelectrode AC Amplifier; A-M Systems, Sequim, WA, USA), filtered (band pass, 10-10 $000 \mathrm{~Hz}$ ) and sent (sampling rate, $20 \mathrm{kHz}$ ) to a computer running Labchart6 recording software (ADInstruments). The preparation was stabilized for 1 hour before any experimental procedure was started. Spontaneous MAN activity was observed for 30 seconds in 2 groups. In order to test the response of nerves to chemical or mechanical stimulus and confirm the nerve recorded as the afferent as required for evidence, perfusing with hydrochloric acid (10 $\mathrm{mM})$ or increasing internal pressure $\left(20 \mathrm{~cm} \mathrm{H}_{2} \mathrm{O}\right)$ in the intestine were performed, and mesenteric nerve activity was recorded for 30 seconds.

\section{Obtaining Mucosal Mediators From Different Bowel Segments}

Bowel segments (30 $\mathrm{mm}$ in length) were obtained from the ileums, proximal colons, and distal colons of guinea pigs from the control and PI-IBS groups. Mucosa was scraped and divided into four equal parts, and then immersed in Eppendorf tubes containing $1 \mathrm{ml}$ of Krebs solution, that was continuously oxygenated $\left(95 \% \mathrm{O}_{2}+5 \% \mathrm{CO}_{2}\right)$ at $37^{\circ} \mathrm{C}$. To investigate whether mast cells influenced mesenteric nerve activity, the scraped mucosa from 
the different bowel segments in the PI-IBS group were treated as described above but with the mast cell stabilizer Nasmil in the Krebs solution ( $1 \mathrm{mg} / \mathrm{mL}$, Sigma, St. Louis, MO, USA). In our preliminary experiment, Nasmil had no effect to the mesenteric nerve activity in the perfusion with mucosa extract in the control group. The mucosa and solution were incubated in the Eppendorf tubes for 30 minutes after the mucosa was shredded. All samples were then centrifuged at $200 \mathrm{~g}$ for 10 minutes and $800 \mu \mathrm{L}$ of supernatant from each tube was moved to another tube which was then marked and stored at $-70^{\circ} \mathrm{C}$. Finally, the mucosa from each segment was blotted and weighed. The mucosa weight from each segment had no obvious difference.

\section{Measuring the Effects of Different Mucosal Supernatants on Mesenteric Afferent Nerve Activity}

Bowel segments from another 10 female adult guinea pigs $(330 \pm 50 \mathrm{~g})$ were prepared as described above. After 1 hour of stabilization, about $3.2 \mathrm{~mL}$ of the supernatant from the same segment (four Eppendorf tubes added) was infused into the bowel by a microinjection pump at a flow rate of $10 \mathrm{~mL} / \mathrm{hr}$. The MAN activity was recorded as described above for 10 minutes. Each bowel segment was infused with mucosal supernatant from the ileums, proximal colons, and distal colons of guinea pigs from the control group, PI-IBS group and the mucosa supernatants from PI-IBS pretreated with Nasmil group. Each segment was stimulated with the nine types of mucosal supernatants. The order in which the supernatants were infused into each bowel segment was random. The bowel was washed with Krebs solution between 2 adjacent interventions, and the next supernatant was infused after the afferent nerve returned to baseline.

\section{Statistical Methods}

The inflammation scores of various bowel segments were expressed as median (interquartile range), and the differences among groups were assessed using the Kruskal-Wallis one-way analysis of variance on ranks. The Wilcoxon rank sum test with a Bonferroni correction was used for multiple comparisons. Other data, such as mast cell counts and afferent nerve discharge frequencies were expressed as mean \pm standard deviation. These data were analyzed using the Student $t$ test or analysis of variance (ANOVA) and the least significant difference (LSD) test when required. Statistical analyses were performed with SPSS 18.0 (SPSS Inc, Chicago, IL, USA) and GraphPad Prism 5 software (GraphPad, San Diego, CA, USA) was used for all graph creation. A $P$-value of $<0.05$ was considered statistically significant.

\section{Results}

\section{Mucosal Histopathology}

At the acute phase of $T$. spiralis infection (2 weeks post-infection, PI-2W), obvious lesions and inflammation were present in the whole intestine, with severe hyperemia and swelling on macroscopic observation and serious eosinophilic and neutrophilic infiltration in the lamina propria at the microscopic level. The inflammation scores in the terminal ileum, proximal colon, and distal colon were much higher than those scores in the controls ( $P=0.009,0.007$, and 0.007, respectively; Table). However, at 8 weeks post-infection (PI-8W), the intestinal inflammation almost disappeared, and the intestine seemed normal. No apparent microscopic inflammatory infiltration or interstitial edema was observed in the intestine. The histological scores for the terminal ileum, proximal colon, and distal colon specimens did not differ between the PI-8W group and the controls $(P=$ $0.197,0.572$, and 0.288 , respectively; Table).

\section{Mast Cell Infiltration in Mucosa From Various Bowel Segments}

Mast cells counting in various bowel segments was performed in the PI-IBS group (8weeks after infection) and the control groups. The number of mast cells in the mucosa of the terminal ileum was much higher in the PI-IBS groups than in the control group $(11.24 \pm 0.45$ vs. $8.80 \pm 0.82, P=0.013$; Fig. $1 \mathrm{~A}$, $1 \mathrm{D}$ and $1 \mathrm{G})$. Similarly, the number of mast cells in the mucosa of the proximal colon was significantly higher in the PI-IBS group than in controls $(13.64 \pm 0.71$ vs. $10.57 \pm 1.08, P=0.026$; Fig. $1 \mathrm{~B}, 1 \mathrm{E}$ and $1 \mathrm{G})$. In the mucosa of the distal colon, mast cells also tended to be more numerous in the PI-IBS group than in con-

Table. Inflmmation Scores in H\&E Stained Sections

\begin{tabular}{cccc} 
Groups & Terminal ileum & Proximal colon & Distal colon \\
Control $(\mathrm{n}=5)$ & $0.10(0.00-0.25)$ & $0.00(0.00-0.20)$ & $0.00(0.00-0.10)$ \\
PI-2W $(\mathrm{n}=5)$ & $2.90(2.65-3.00)^{\mathrm{a}}$ & $2.80(2.45-2.80)^{\mathrm{a}}$ & $2.70(2.60-2.85)^{\mathrm{a}}$ \\
PI-8W $(\mathrm{n}=5)$ & $0.30(0.10-0.40)$ & $0.10(0.10-0.30)$ & $0.10(0.00-0.35)$ \\
\hline
\end{tabular}

${ }^{a}$ Represent statistically significant from control (Kruskal-Wallis one-way analysis of variance on ranks, Bonferroni-corrected $P<0.05 / 3$ ).

PI-2W, 2 weeks post infection; PI-8W, 8 weeks post infection.

Data are presented as median (interquartile range). 

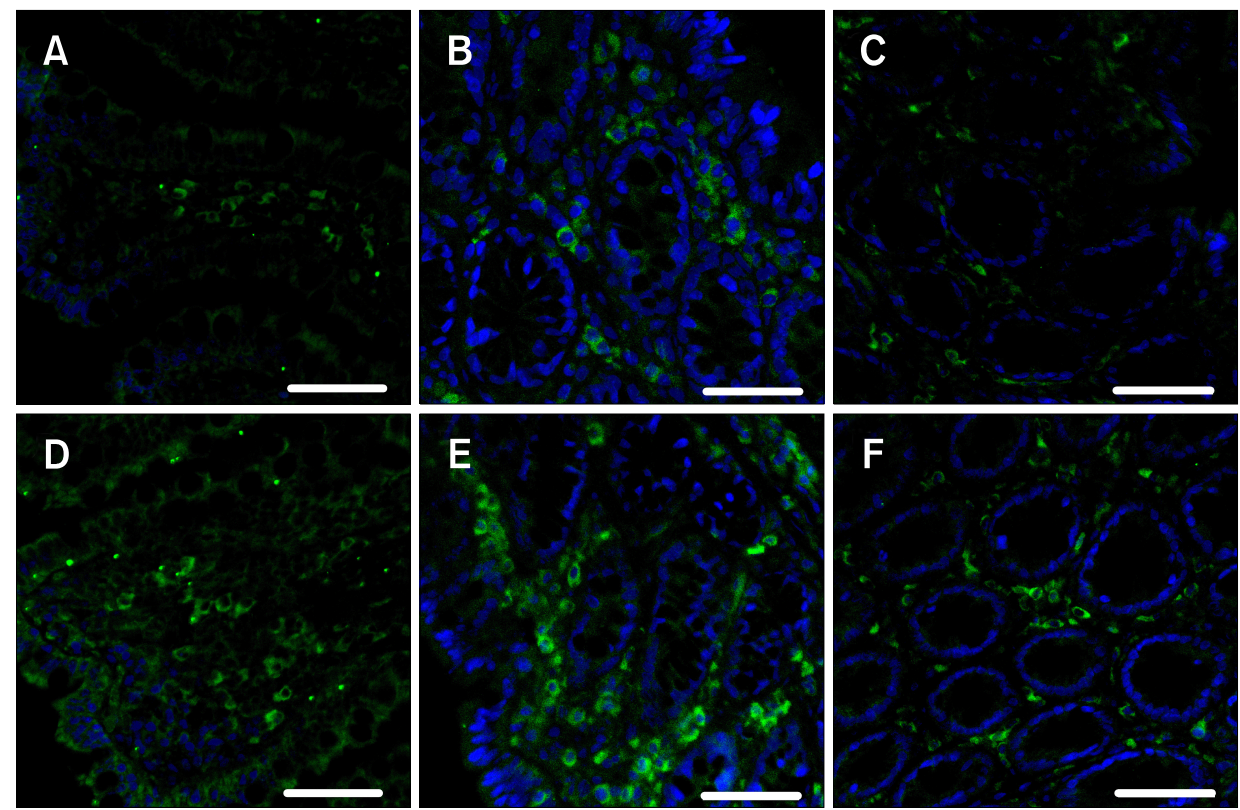

Terminal ileum

Proximal colon

$\mathbf{G}$

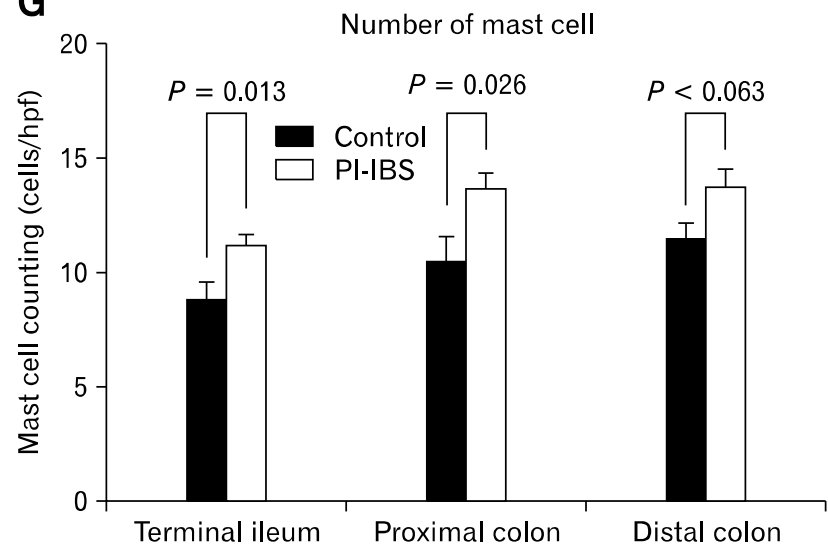

trols, but the difference between the groups was not statistically significant (13.74 \pm 0.77 vs $11.50 \pm 0.59, P=0.063$; Fig. $1 \mathrm{C}$, $1 \mathrm{~F}$, and $1 \mathrm{G})$.

\section{Spontaneous Mesenteric Nerve Activity}

The spontaneous discharge frequency of MANs was 75.98 $\pm 4.71 \mathrm{~Hz}$ in the PI-IBS group, which was significantly higher than that in the control group $(31.36 \pm 4.71 \mathrm{~Hz}, P<0.001$; Fig. 2).

\section{lleum Mucosal Supernatant Excited Mesenteric Nerve}

The average discharge frequency of the MANs was $41.01 \pm$ 7.6 $\mathrm{Hz}$ when perfused with ileum mucosal supernatant from the PI-IBS group, which was significantly higher than at the baseline

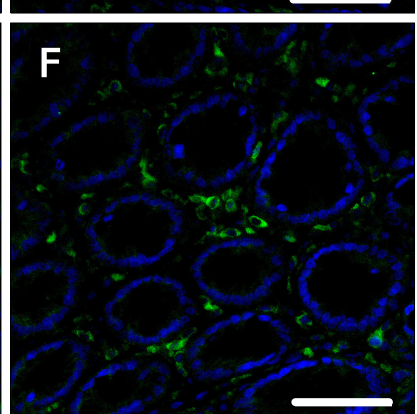

Distal colon

Figure 1. Immunofluorescence staining for tryptase (mast cells) in the terminal ileum, proximal colon, and distal colon sections of control groups (A, B, and $\mathrm{C}$, respectively) and PI-IBS groups (D, E , and F, respectively) at $\times 600$ magnification. Mucosal mast cell infiltration increased significantly in post-infectious irritable bowel syndrome (PI-IBS) models compared with controls. (G) The mean number of mast cell in the terminal ileum and proximal colon was higher in PIIBS than in controls $(P<0.05)$, while there was no significant difference in distal colon between the 2 groups ( $P=0.063)$. Green indicates tryptase, blue indicates DAPI, and the scale bar in A-F is 50 $\mu \mathrm{m}$. hpf, high-power field.

(24.24 $\pm 7.0 \mathrm{~Hz}, P=0.001$; Fig. 3B). However, the discharge frequency was $26.55 \pm 0.67 \mathrm{~Hz}$ when perfused with ileum mucosal supernatant from the control group, and did not significantly differ from the activity level at baseline $(25.93 \pm 2.37$ $\mathrm{Hz}, P=0.666$; Fig. 3A). Ileum mucosal supernatant from the PI-IBS group also induced much more intensive MANs firing than that from the control group $(41.01 \pm 7.6 \mathrm{~Hz}$ vs. $26.55 \pm$ $0.67 \mathrm{~Hz}, P=0.001)$. In addition, ileum mucosal supernatant from the PI-IBS group pretreated with Nasmil led to a mean frequency of $32.71 \pm 2.52 \mathrm{~Hz}$, which was higher than the frequency at baseline $(25.98 \pm 1.42 \mathrm{~Hz}, P=0.015$; Fig. 3C) and higher than the frequency induced by ileum mucosal supernatant from the control group $(26.55 \pm 0.67 \mathrm{~Hz}, P=0.023)$, but significantly lower than the frequency induced by supernatant from the PI-IBS group without Nasmil $(41.01 \pm 7.6 \mathrm{~Hz}, P=0.030$; 
A

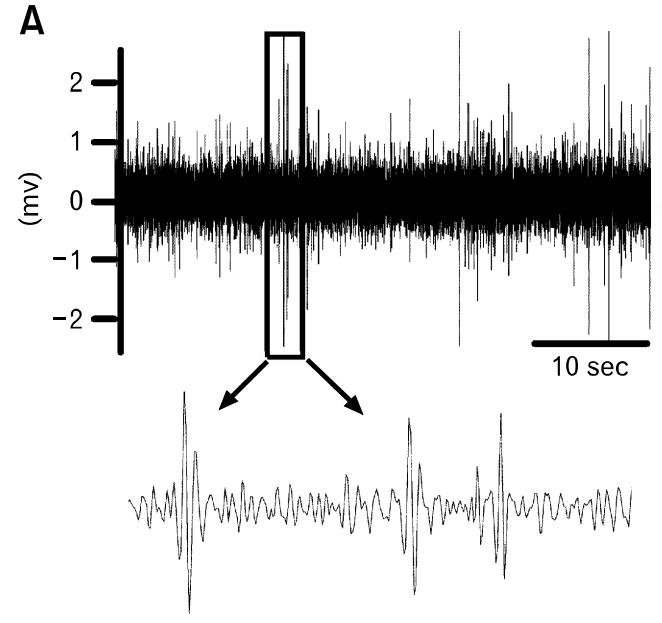

B

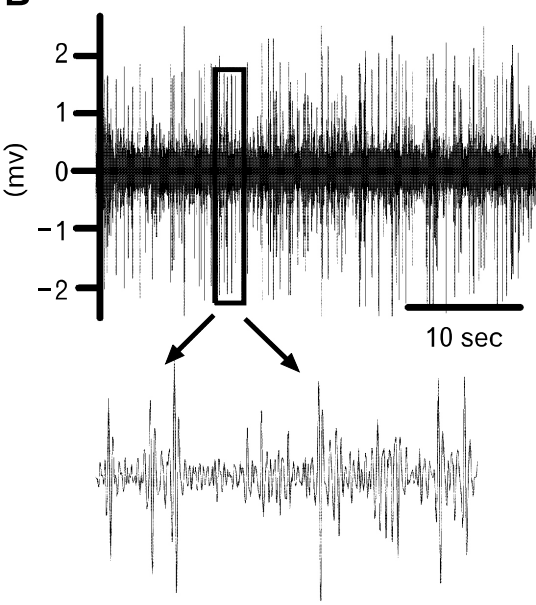

C

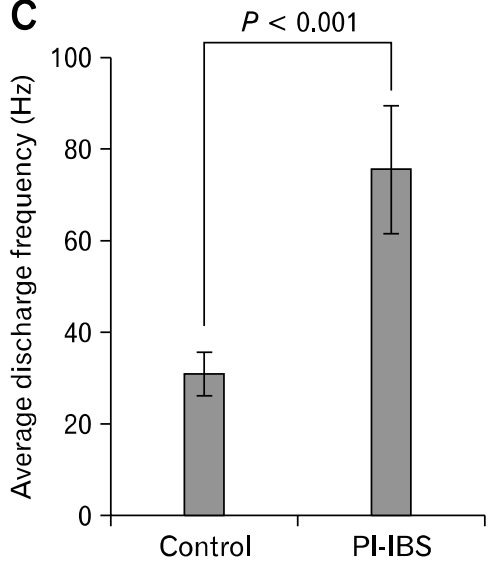

Figure 2. Spontaneous mesenteric nerve activity from the control group (A) and the post-infectious irritable bowel syndrome (PI-IBS) group (B). The spontaneous discharge frequency of mesenteric nerves was enhanced in PI-IBS group compared with the controls $(P<0.001)(\mathrm{C})$.

A
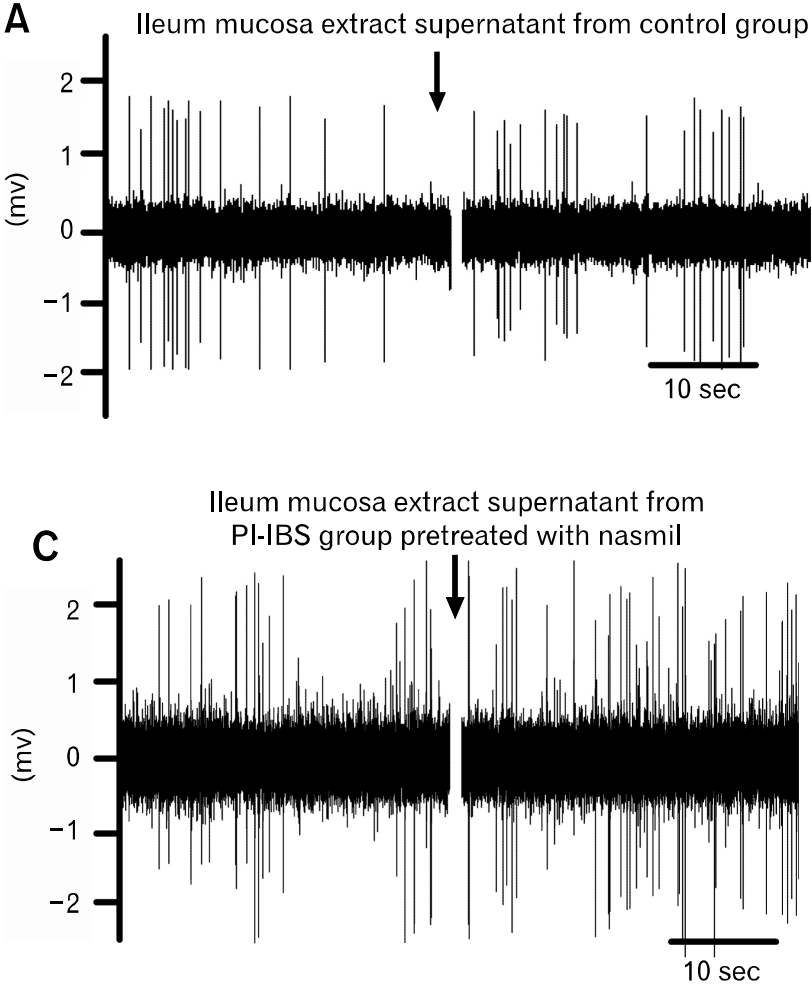

B

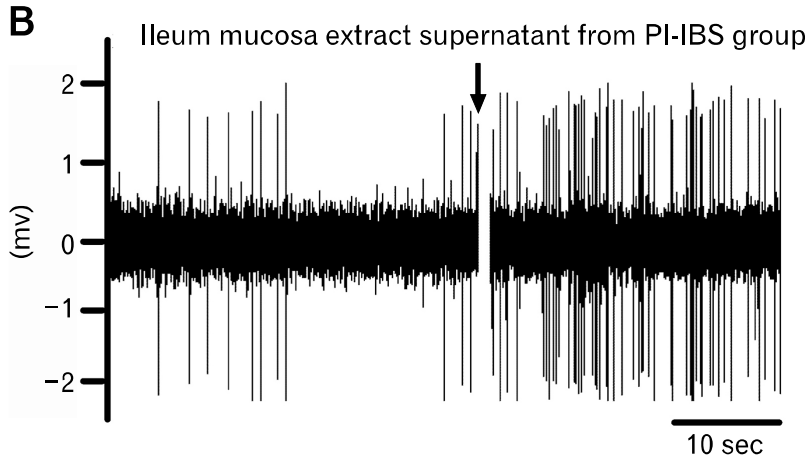

D $\square$ Baseline $\square$ After perfusion

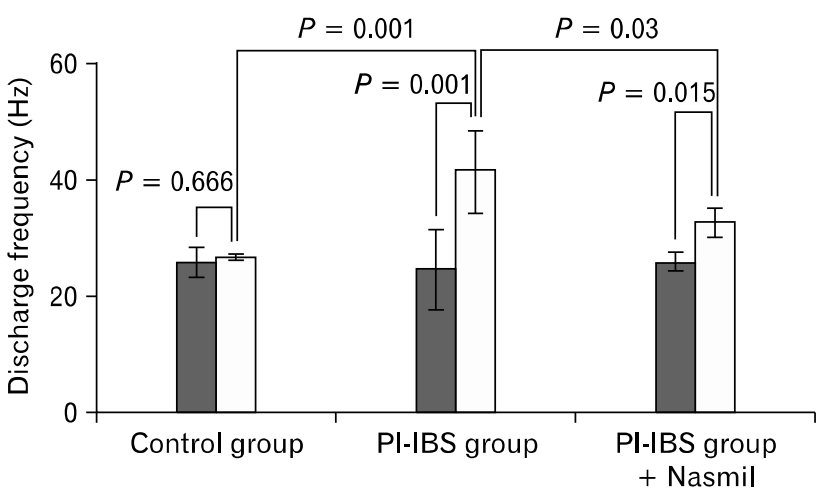

Figure 3. Effects of terminal ileum mucosal supernatant from the control group (A) and the post-infectious irritable bowel syndrome (PI-IBS) group (B), and effects of ileum mucosal supernatant from the PI-IBS group pretreated with Nasmil (C) on mesenteric nerve firing. (D) Discharge frequency of mesenteric nerves at baseline and after perfusion of ileum mucosal supernatant from control, PI-IBS, and PI-IBS + Nasmil groups. 
Fig. 3D).

\section{Proximal Colon Mucosal Supernatant Excited Mesenteric Nerve}

The average discharge frequency of the MANs was $45.9 \pm$ 11.2 $\mathrm{Hz}$ when perfused with proximal colon mucosal supernatant from the PI-IBS group, which was much higher than the frequency at baseline $(25 \pm 9.35 \mathrm{~Hz}, P<0.001$; Fig. 4B). However, the discharge frequency was $30.8 \pm 6.9 \mathrm{~Hz}$ when perfused with proximal colon mucosal supernatant from the control group and did not significantly differ from that at baseline (27.2 $\pm 6.9 \mathrm{~Hz}, P=0.417$; Fig. 4A). Proximal colon mucosal supernatant from the PI-IBS group excited the MANs significantly more than that from the control group $(45.9 \pm 11.2 \mathrm{~Hz}$ vs. 30.88 $\pm 6.92 \mathrm{~Hz}, P=0.002$ ). Moreover, the mean frequency was $30.94 \pm 4.44 \mathrm{~Hz}$ when perfused with proximal colon mucosal supernatant from the PI-IBS group pretreated with Nasmil, which was higher than that at baseline $(26.14 \pm 2.15 \mathrm{~Hz}, P=$ 0.010 ; Fig. 4C) but was not significantly different from that of MANs perfused with supernatant from the controls $(30.88 \pm$ $6.92 \mathrm{~Hz}, P=0.866)$, and was significantly lower than that when perfused with supernatant from the PI-IBS group without Nasmil (45.9 $\pm 11.2 \mathrm{~Hz}, P=0.002$; Fig. 4D).

\section{Distal Colon Mucosal Supernatant Excited Mesenteric Nerve}

The mean discharge frequency of the MANs was 29.47士 $6.13 \mathrm{~Hz}$ when perfused with distal colon mucosal supernatant from the control group, which was not significantly different from that at baseline $(28.18 \pm 6.71 \mathrm{~Hz}, P=0.694$; Fig. 5A). However, Mucosal supernatant extracted from the distal colon of the PI- IBS group induced significant nerve firing $(48.25 \pm 9.7$ $\mathrm{Hz}$ vs. $25.43 \pm 7.6 \mathrm{~Hz}$ at baseline, $P<0.001$; Fig. $5 \mathrm{~B}$ ) that was more intensive than that induced by distal colon mucosal super-
A

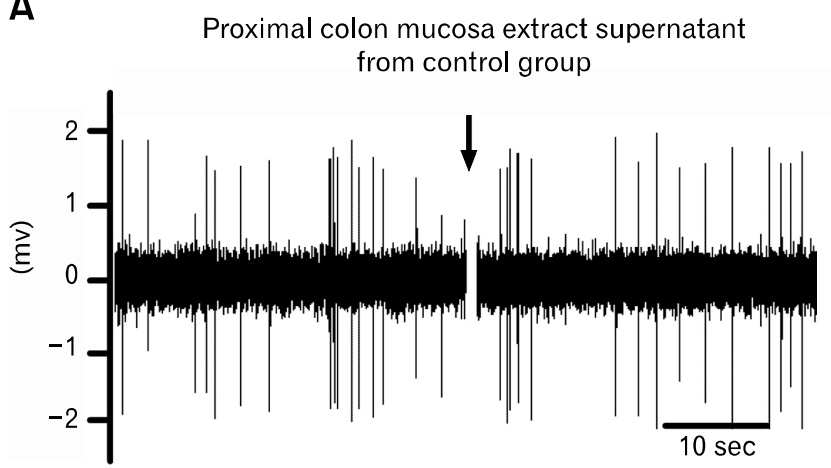

C

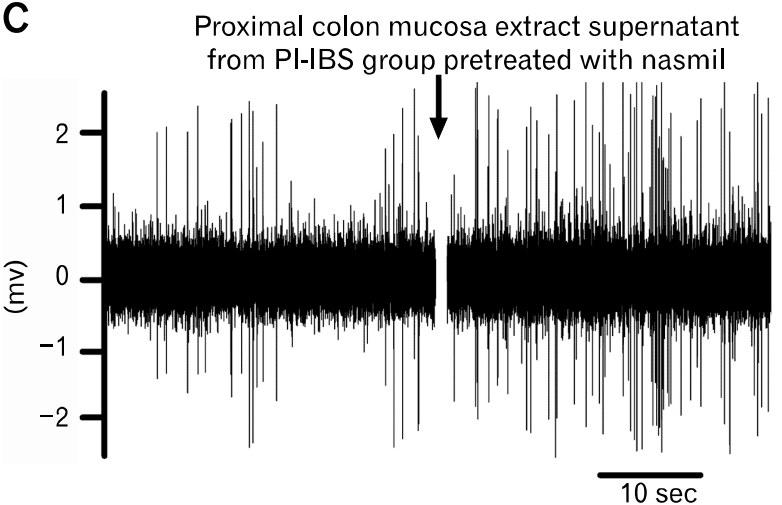

B Proximal colon mucosa extract supernatant

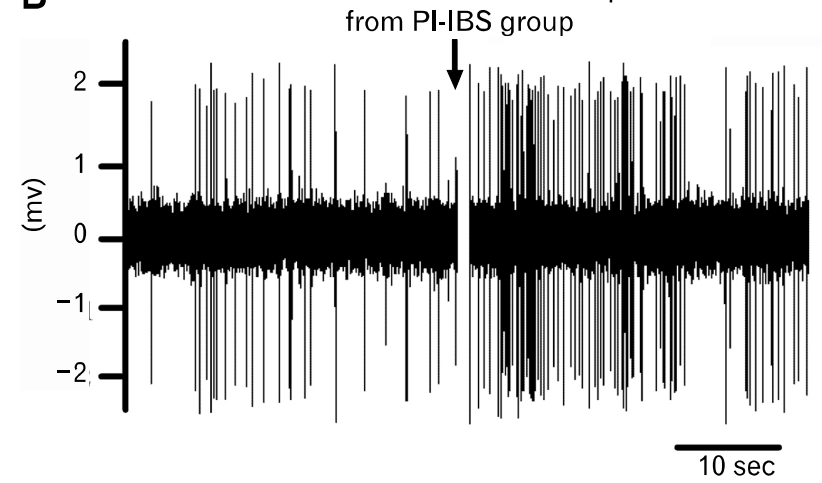

D $\square$ Baseline $\square$ After perfusion

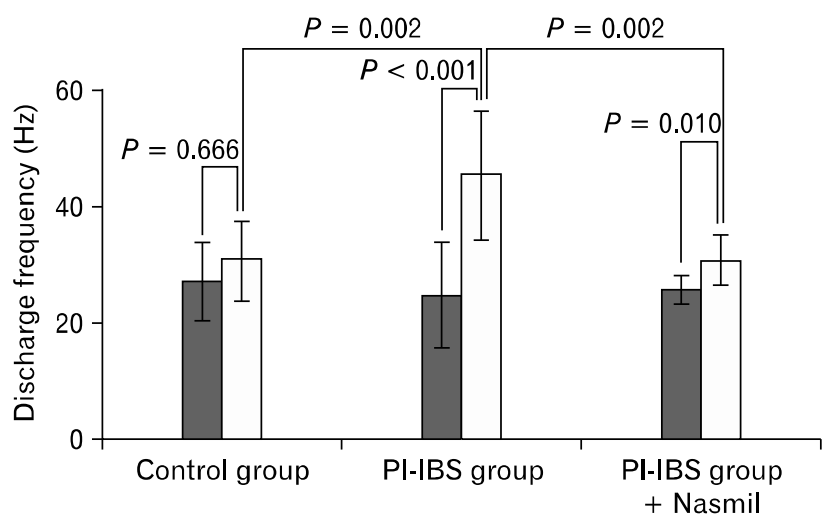

Figure 4. Effects of proximal colon mucosal supernatant from the control group (A) and the post-infectious irritable bowel syndrome (PI-IBS) group (B), and effects of proximal colon mucosal supernatant from the PI-IBS group pretreated with Nasmil (C) on mesenteric nerve firing. (D) Discharge frequency of mesenteric nerves at baseline and after perfusion of proximal colon mucosal supernatant from control, PI-IBS and PI-IBS + Nasmil groups. 
A

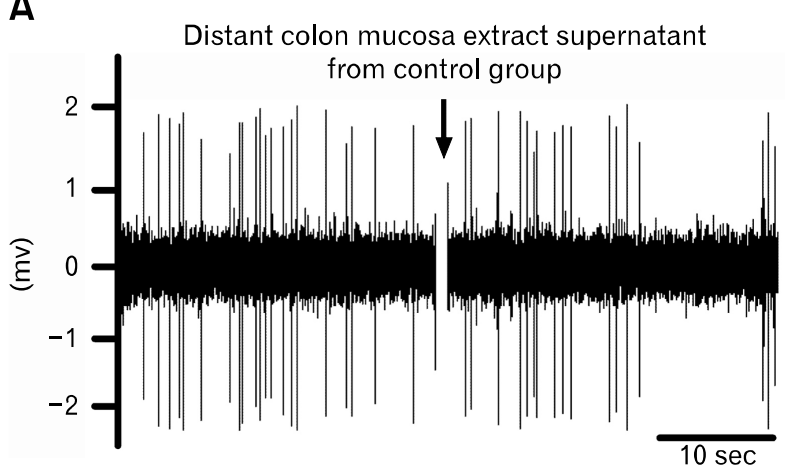

C

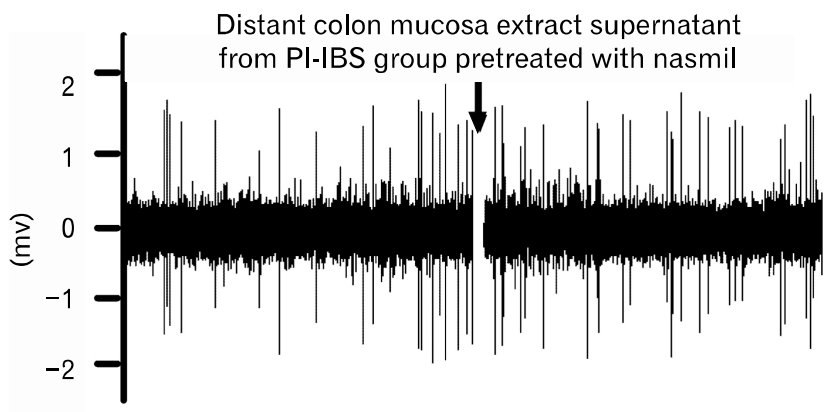

B

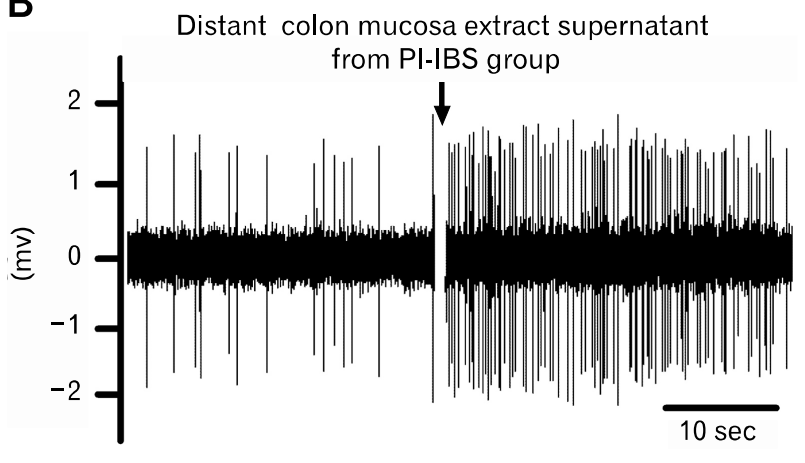

D $\square$ Baseline $\square$ After perfusion

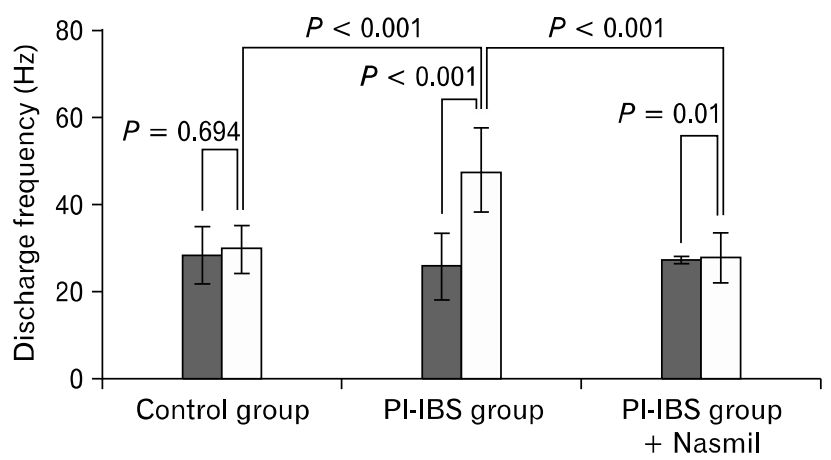

Figure 5. Effects of terminal distal colon mucosal supernatant from the control group (A) and the stabilizer Nasmil (PI-IBS) group (B), and effects of distal colon mucosal supernatant from the PI-IBS group pretreated with Nasmil (C) on mesenteric nerve firing. (D) Discharge frequency of mesenteric nerves at baseline and after perfusion of distal colon mucosal supernatant from control, PI-IBS, and PI-IBS + Nasmil groups.

natant from the controls $(48.25 \pm 9.7 \mathrm{~Hz}$ vs. $29.47 \pm 6.13 \mathrm{~Hz}$ $P<0.001)$. Furthermore, when the PI-IBS group was pretreated with Nasmil, the nerve firing induced by the distal colon mucosal supernatant was weaker than that induced by the supernatant from the PI-IBS group without Nasmil $(27.51 \pm 5.83 \mathrm{~Hz}$ vs. $48.25 \pm 9.7 \mathrm{~Hz}, P<0.001)$. The MAN firing induced by the supernatant from the PI-IBS group pretreated with Nasmil did not differ significantly from that at baseline $(26.75 \pm 1.18$ $\mathrm{Hz}, P=0.722$; Fig. 5C) and that induced by the supernatant from the control group $(29.47 \pm 6.13 \mathrm{~Hz}, P=0.754$; Fig. 5D).

\section{Discussion}

In this study, we observed that the electrical activity of MANs was enhanced significantly in healthy guinea pig bowel segments perfused with mucosal supernatant from various bowel segments of guinea pigs with PI-IBS compared with mucosal supernatant from bowels of guinea pigs without PI-IBS. We also observed that the mucosal mast cells were more numerous in mucosa from various segments from the PI-IBS group than in mucosa from the controls and that the mast cell stabilizer Nasmil alleviated the exciting effects of the mucosal supernatant from the PI-IBS group. These findings indicate that mast cell-mediated MAN activation plays an important role in the pathogenesis of PI-IBS.

Visceral hypersensitivity is a key pathophysiologic mechanism of IBS and PI-IBS. ${ }^{25}$ Compared with healthy people, IBS patients, including those with PI-IBS, felt more pain in response to rectal distension with the same pressure. ${ }^{26,27}$ Animal models for PI-IBS also manifested persistent hyperalgesia to mechanical stimulation. ${ }^{28}$ As the primary pathway from the gut to the brain, MANs play key roles in visceral sensation. There are abundant chemical and mechanical sensitive nerve endings distributed in the gut wall, which are key for maintaining the physiologic functions of the gut and participate in the pathogenesis of symptoms such as abdominal pain. It was confirmed that some receptors or 
ion channels such as non-selective cation channels containing VR1, acid-sensing ion channel (ASIC), and G-protein receptors increased in patients and animal models with IBS. ${ }^{29}$ Previous studies have shown that visceral afferent nerves are involved in visceral hypersensitivity. Pelvic afferents were hyper-mechanosensitive in trinitrobenzene sulfonic acid (TNBS)-induced colitis in rodents, which was regarded as a model of some aspects of IBS. ${ }^{30}$ Intestinal afferent nerves manifested hyper-chemosensitivity in a PI-IBS model infected with Nippostrongylus brasiliensis larvae. ${ }^{31}$ In this study, we found that the spontaneous discharge frequency of MANs was significantly enhanced in a PI-IBS model infected with T. spiralis. We also observed that MANs were more sensitive not only to physiologic and noxious pressure stimulation but also to acid stimulation in PI-IBS models with $T$. spiralis infection (results were not presented in this paper). These findings suggest that MANs remain in a sensitized state in PI- IBS and may be related to visceral hypersensitivity in PI-IBS.

To date, abundant evidence shows that low-grade immune activation exists in the gut in PI-IBS, although PI-IBS has been recognized as a non-organic disease. ${ }^{32}$ Consistent with previous studies, we observed that the histologic manifestation of PI-IBS tended to be normal in animal models. A series of studies supported this immune dysfunction in IBS patients or animal models of IBS; for example, an imbalance between pro-inflammatory cytokines (TNF- $\alpha$, IL-1 $\beta$, IL-6, etc) and anti-inflammatory cytokines (IL-10, etc) with a Thl/Th2 shift in the gut was observed in PI-IBS. ${ }^{33}$ The mucosal immune disorder was related to increased visceral pain. Cytokines such as IL-1 $\beta$ excited ENS neurons related to visceral hypersensitivity in IBS and inflammatory bowel disease. ${ }^{34}$ We observed the effect of mucosal supernatant from the ileum, proximal colon, and distal colon from guinea pigs with PI-IBS on MAN activity and found that mucosal mediators from each of those bowel segments enhanced the nerve firing, indicating that abnormal secretions exist throughout the whole intestinal mucosa in PI-IBS and are able to excite sensory neural endings in some way. This inappropriate information in the gut was transmitted to the brain through MANs and may ultimately arouse the abdominal pain or perception of discomfort experienced by IBS patients.

Increasing evidence favoring an important role of mast cells in the pathophysiology of IBS has been reported. ${ }^{35,36}$ Several studies have shown that the number of mast cells and/or the spontaneous release of mediators such as proteases, histamine, and serotonin is increased in the gut of IBS. ${ }^{36,37}$ In our study, we confirmed a significant increase in mast cell infiltration in the ileum and proximal colon and a trend of increased mast cell infiltration in the distal colon in the PI-IBS group compared with controls. Mast cells have been widely studied in IBS patients and several animal models; for example, mast cell tryptase increased epithelial permeability and intestinal barrier dysfunction in a PAR2-mediated manner. ${ }^{38}$ Lee et $\mathrm{al}^{39}$ found that rectal mucosal mast cell count was significantly correlated with intestinal permeability in IBS-D patients, which indicated mucosal mast cells to play an important role for increased permeability in patients with IBS-D. As an important component of the innate immune system, mast cells also play a significant role in immune disorders in the gut and especially in the neuro-immune interaction that may be involved in the development of visceral hyperalgesia. Previous study by $\mathrm{Yu}$ et $\mathrm{al}^{40}$ demonstrated that mast cell activation induced long-lasting mechanical hypersensitivity of nodose C-fibers in the guinea pig esophagus and mast cell trypase played an important role in this sensitization process. Moreover, there was an increasing proportion of mast cells in proximity to nerve fibers that correlated with the severity of abdominal pain perception in IBS. $^{37} \mathrm{We}$ speculated that mast cells and their mediators were the main factors in the mucosal supernatant responsible for MAN activation. Treatment with ketotifen, a mast cell stabilizer, increased the threshold of discomfort for IBS patients with visceral hypersensitivity and improved IBS symptoms such as abdominal pain, bloating and diarrhea. ${ }^{35}$ Although our study did not identify the particular substances that excited MANs, our data indicated that the mast cell stabilizer Nasmil could significantly inhibit the exciting effect of mucosal supernatant from various intestinal segments, especially the distal colon, on mesenteric nerves. These findings suggest that mast cell mediators could be the key components of these mucosal supernatants' effects. All of the above strongly points towards an important role off mast cells in the abnormal MAN activation and visceral hypersensitivity noted in PI-IBS.

In summary, the spontaneous electrical activity of MANs increased significantly in PI-IBS, and MAN hypersensitivity was involved in PI-IBS. In our attempt to elucidate the peripheral mechanism of visceral hypersensitivity in PI-IBS, we confirmed that abnormal mucosal mediators from various bowel segments in PI-IBS models led to excitation of MANs at least partly in a mast cell-dependent manner, indicating that mast cell-dependent MAN activation plays an important role in the pathogenesis of PI-IBS. Further studies need to be done to identify the particular factors and receptors in the intestinal mucosa that lead to this MAN activation, which may provide therapeutic targets for 
PI-IBS.

\section{Acknowledgements}

We are grateful for the advices provided by Dr. Xuelian Xiang and Dr. Yun Yan in the protocol design.

\section{References}

1. Saito YA, Schoenfeld P, Locke GR 3rd. The epidemiology of irritable bowel syndrome in North America: a systematic review. Am J Gastroenterol 2002; 97:1910-1915.

2. Spiller RC. Postinfectious irritable bowel syndrome. Gastroenterology 2003;124:1662-1671.

3. Spiller R, Garsed K. Postinfectious irritable bowel syndrome. Gastroenterology 2009;136:1979-1988.

4. Spiller RC, Jenkins D, Thornley JP, et al. Increased rectal mucosal enteroendocrine cells, $\mathrm{T}$ lymphocytes, and increased gut permeability following acute Campylobacter enteritis and in post-dysenteric irritable bowel syndrome. Gut 2000;47:804-811.

5. Marshall JK, Thabane M, Garg AX, et al. Intestinal permeability in patients with irritable bowel syndrome after a waterborne outbreak of acute gastroenteritis in Walkerton, Ontario. Aliment Pharmacol Ther 2004;20:1317-1322.

6. Wang LH, Fang XC, Pan GZ. Bacillary dysentery as a causative factor of irritable bowel syndrome and its pathogenesis. Gut 2004; 53:1096-1101.

7. Yang X, Sheng L, Guan Y, Qian W, Hou X. Synaptic plasticity: the new explanation of visceral hypersensitivity in rats with trichinella spiralis infection? Dig Dis Sci 2009;54:937-946.

8. Spiller R, Lam C. An update on post-infectious irritable bowel syndrome: role of genetics, immune activation, serotonin and altered microbiome. J Neurogastroenterol Motil 2012;18:258-268.

9. Elsenbruch S. Abdominal pain in irritable bowel syndrome: a review of putative psychological, neural and neuro-immune mechanisms. Brain Behav Immun 2011;25:386-394.

10. Kanazawa M, Hongo M, Fukudo S. Visceral hypersensitivity in irritable bowel syndrome. J Gastroenterol Hepatol 2011;26(suppl 3): 119-121.

11. Ibeakanma C, Ochoa-Cortes F, Miranda-Morales M, et al. Braingut interactions increase peripheral nociceptive signaling in mice with postinfectious irritable bowel syndrome. Gastroenterology 2011;141: 2098-2108, e5.

12. Aizawa E, Sato Y, Kochiyama T, et al. Altered cognitive function of prefrontal cortex during error feedback in patients with irritable bowel syndrome, based on FMRI and dynamic causal modeling. Gastroenterology 2012;143:1188-1198.

13. van Orshoven NP, Andriesse GI, van Schelven LJ, Smout AJ, Akkermans LM, Oey PL. Subtle involvement of the parasympathetic nervous system in patients with irritable bowel syndrome. Clin Auton Res 2006;16:33-39.

14. Gupta V, Sheffield D, Verne GN. Evidence for autonomic dysregulation in the irritable bowel syndrome. Dig Dis Sci 2002;47:17161722.
15. Grundy D. Neuroanatomy of visceral nociception: vagal and splanchnic afferent. Gut 2002;51(suppl 1):i2-i5.

16. Hillsley K, Grundy D. Sensitivity to 5-hydroxytryptamine in different afferent subpopulations within mesenteric nerves supplying the rat jejunum. J Physiol 1998;509(Pt 3):717-727.

17. Gwee KA. Post-infectious irritable bowel syndrome, an inflammationimmunological model with relevance for other IBS and functional dyspepsia. J Neurogastroenterol Motil 2010;16:30-34.

18. Buhner S, Schemann M. Mast cell-nerve axis with a focus on the human gut. Biochim Biophys Acta 2012;1822:85-92.

19. Kraneveld AD, Rijnierse A, Nijkamp FP, Garssen J. Neuro-immune interactions in inflammatory bowel disease and irritable bowel syndrome: future therapeutic targets. Eur J Pharmacol 2008;585: 361-374.

20. Barbara G, Wang B, Stanghellini V, et al. Mast cell-dependent excitation of visceral-nociceptive sensory neurons in irritable bowel syndrome. Gastroenterology 2007;132:26-37.

21. Bercík P, Wang L, Verdú EF, et al. Visceral hyperalgesia and intestinal dysmotility in a mouse model of postinfective gut dysfunction. Gastroenterology 2004;127:179-187.

22. Jardí F, Fernández-Blanco JA, Martínez V, Vergara P. Plasticity of dorsal root ganglion neurons in a rat model of post-infectious gut dysfunction: potential implication of nerve growth factor. Scand J Gastroenterol 2014;49:1296-1303.

23. Al-Chaer ED, Kawasaki M, Pasricha PJ. A new model of chronic visceral hypersensitivity in adult rats induced by colon irritation during postnatal development. Gastroenterology 2000;119:1276-1285.

24. Keating C, Beyak M, Foley S, et al. Afferent hypersensitivity in mouse model of post-inflammatory gut dysfunction: role of altered serotonin metabolism. J Physiol 2008;586(Pt 18):4517-4530.

25. Azpiroz F, Bouin M, Camilleri M, et al. Mechanisms of hypersensitivity in IBS and functional disorders. Neurogastroenterol Motil 2007;19:62-88.

26. Ludidi S, Conchillo JM, Keszthelyi D, et al. Rectal hypersensitivity as hallmark for irritable bowel syndrome: defining the optimal cutoff. Neurogastroenterol Motil 2012;24:729-733, e345-e346.

27. Vergnolle N. Postinflammatory visceral sensitivity and pain mechanisms. Neurogastroenterol Motil 2008;20(suppl 1):73-80.

28. Ibeakanma C, Miranda-Morales M, Richards M, et al. Citrobacter rodentium colitis evokes post-infectious hyperexcitability of mouse nociceptive colonic dorsal root ganglion neurons. J Physiol 2009; 587(Pt14):3505-3521.

29. McMahon SB. Sensitisation of gastrointestinal tract afferents. Gut 2004;53(suppl 2):ii13-ii15.

30. Hughes PA, Brierley SM, Martin CM, Brookes SJ, Linden DR, Blackshaw LA. Post-inflammatory colonic afferent sensitisation: different subtypes, different pathways and different time courses. Gut 2009;58:1333-1341.

31. Aerssens J, Hillsley K, Peeters PJ, et al. Alterations in the brain-gut axis underlying visceral chemosensitivity in Nippostrongylus brasiliensis-infected mice. Gastroenterology 2007;132:1375-1387.

32. Ghoshal UC, Ranjan P. Post-infectious irritable bowel syndrome: the past, the present and the future. J Gastroenterol Hepatol 2011; 26(suppl 3):94-101.

33. Chen J, Zhang Y, Deng Z. Imbalanced shift of cytokine expression between $T$ helper 1 and T helper 2 (Th1/Th2) in intestinal mucosa of 
patients with post-infectious irritable bowel syndrome. BMC Gastroenterol 2012;12:91-96.

34. Tjwa ET, Bradley JM, Keenan CM, Kroese AB, Sharkey KA. Interleukin-1beta activates specific populations of enteric neurons and enteric glia in the guinea pig ileum and colon. Am J Physiol Gasterointest Liver Physiol 2003;285:G1268- G1276.

35. Klooker TK, Braak B, Koopman KE, et al. The mast cell stabiliser ketotifen decreases visceral hypersensitivity and improves intestinal symptoms in patients with irritable bowel syndrome. Gut 2010;59: 1213-1221.

36. Yang CQ, Wei YY, Zhong CJ, Duan LP. Essential role of mast cells in the visceral hyperalgesia induced by $\mathrm{T}$. spiralis infection and stress in rats. Mediators Inflamm 2012;2012:294070.

37. Barbara G, Stanghellini V, De Giorgio R, et al. Activated mast cells in proximity to colonic nerves correlate with abdominal pain in irritable bowel syndrome. Gastroenterology 2004;126:693-702.

38. Fernández-Blanco JA, Hollenberg MD, Martínez V, Vergara P. PAR-2-mediated control of barrier function and motility differs between early and late phases of postinfectious gut dysfunction in the rat. Am J Physiol Gastrointest Liver Physiol 2013;304:G390-G400.

39. Lee H, Park JH, Park DI, et al. Mucosal mast cell count is associated with intestinal permeability in patients with diarrhea predominant irritable bowel syndrome. J Neurogastroenterol Motil 2013;19: 244-250.

40. Yu S, Gao G, Peterson BZ, Ouyang A. TRPA1 in mast cell activation-induced long-lasting mechanical hypersensitivity of vagal afferent C-fibers in guinea pig esophagus. Am J Physiol Gastrointest Liver Physiol 2009;297:G34-G42. 